\title{
Simultaneous penile prosthesis and male sling/artificial urinary sphincter
}

\begin{abstract}
Dominic Lee ${ }^{1}$, Claudio Romero ${ }^{2}$, Frances Alba $^{2}$, O Lenaine Westney ${ }^{1}$ and Run Wang ${ }^{1,2}$
Erectile dysfunction (ED) and stress urinary incontinence (SUI) from urethral sphincteric deficiency is not an uncommon problem. The commonest etiology is intervention for localized prostate cancer and/or radical cystoprostatectomy for muscle invasive bladder cancer. Despite advances in surgical technology with robotic assisted laparoscopic prostatectomy and nerve sparing techniques, the rates of ED and SUI remain relatively unchanged. They both impact greatly on quality of life domains and have been associated with poor performance outcomes. Both the artificial urinary sphincter and penile prosthesis are gold standard treatments with proven efficacy, satisfaction and durability for end-stage SUI and ED respectively. Simultaneous prosthesis implantation for concurrent conditions has been well described, mostly in small retrospective series. The uptake of combination surgery has been slow due in part to technical demands of the surgery and to an extent, a heightened anxiety over potential complications. This paper aims to discuss the technical aspect of concurrent surgery for both disease entity and the current published outcomes of the various surgical techniques with this approach.
\end{abstract}

Asian Journal of Andrology (2013) 15, 10-15; doi:10.1038/aja.2012.115; published online 3 December 2012

Keywords: artificial urinary sphincter; dual implants; erectile dysfunction; penile prosthesis; stress urinary incontinence

\section{INTRODUCTION}

Combination surgery

Both erectile dysfunction (ED) and stress urinary incontinence (SUI) are serious post-operative adverse events in men undergoing surgical intervention for both prostate and bladder cancer. Together, these conditions contribute significantly to the decrease in the post-operative quality of life scores leading to general dissatisfaction. The estimated mean potency rate in contemporary radical prostatectomy series has been estimated at 19\% (range: 11\%-40\%) regardless of operative technique. ${ }^{1}$ From the Prostate Cancer Outcomes Study (PCOS), the estimated incontinence rate has been variably estimated between $3 \%$ and $74 \%$, due partly to lack of standardization for the definition of continence. The reported quality of life impairment of SUI and ED is $13 \%$ and $46 \%$, respectively. ${ }^{2}$

For the management of severe SUI, the artificial urinary sphincter (AUS) (AMS 800; American Medical Systems, Minnetonka, MN, USA) is the gold standard. ${ }^{3,4}$ However for those with mild to moderately severe SUI, the Advance ${ }^{\mathrm{TM}}$ male transobturator sling (American Medical Systems) remains a popular choice. ${ }^{5}$ Recent 3-year data from a multi-institutional study have confirmed efficacy and durability of effect with regards to the male sling. ${ }^{6}$

The inflatable penile prosthesis (IPP) is the standard of care for men with medically refractory ED. It has consistently performed well with high patient satisfaction and prosthesis survival rates. ${ }^{7}$ Modifications to the prosthesis components, especially with antibiotic retardant coating, has translated to significant decreases in post-operative infection complications. ${ }^{8}$ In recent times, the trans-scrotal technique for prosthesis placement by Wilson et al. ${ }^{9}$ has further simplified the implantation approach and offers concurrent prosthesis insertion via a single incision to address both SUI and ED.

Despite obvious advantages, including cost and time savings to the patient, adoption of simultaneous prosthesis placement has been slow. Initial studies with earlier penile and urinary sphincter models reported higher infection and mechanical failure rates resulting in concerns over these complications contributing to skepticisms with this surgical approach. ${ }^{10}$ To date, there is a paucity of published data on outcomes for combination surgery for management of both ED and male SUI. The majority are single institution case series reporting on IPP with AUS with small patient numbers and short-term followup. ${ }^{11}$ Recently, the senior author of this paper published his series on combination surgery with IPP and male sling with promising results. ${ }^{12}$ Other evolving SUI products such as the adjustable balloon devices (Pro-ACT ${ }^{\mathrm{TM}}$; Uromedica Inc., MN, USA), currently pending FDA approval, will also be briefly reviewed in consideration for potential use in combination surgery. ${ }^{13}$

\section{Patient selection}

Depending on institution referral patterns and available expertise, most patients are assessed independently for ED and SUI following failure of conservative management. The evaluation process consists of: (i) clinical history to elicit etiology (surgery, radiotherapy, multimodal therapy), duration of impairment, previous treatment regimes, previous abdominal or genitourinary surgery and comorbidities; (ii) genito-urinary examination for documentation of penile length, 
evidence of penile plaques, scrotal contents and evidence of inguinal hernia. Health of genital skin will be assessed and any evidence of skinrelated infections warrants active treatment prior to prosthetic surgery; (iii) quantification of organ dysfunction severity with pad use and validated questionnaires such as the International Continence Society short form (men) and Sexual Health Inventory for Men score; and (iv) genito-urinary investigations including but not limited to a 3day voiding diary, 24-h pad test, urodynamic study and flexible cystoscopy. Urodynamic testing is used to assess bladder stability, bladder compliance and sphincteric integrity/incompetence. ED work-up, if indicated, includes hormonal profile with testosterone and penile doppler study.

\section{AUS versus male slings}

There is no available consensus detailing the appropriate treatment option to match SUI severity. Clinical trials are lacking comparing AUS versus male slings. To some extent, SUI severity is subjective and personally dependent on the patients' threshold of wetness. Pad use is subjective and may not always reflect severity of incontinence. ${ }^{14}$ Pad weight over $24 \mathrm{~h}$, although cumbersome, is a more objective measure of severity for post-prostatectomy incontinence (PPI) patients. The following has been categorized: $\leqslant 100 \mathrm{gm} / 24 \mathrm{~h}$ as mild, $100-400 \mathrm{gm} / 24 \mathrm{~h}$ as moderate, and $\geqslant 400 \mathrm{gm} / 24 \mathrm{~h}$ as severe SUI. Choice of therapy is a complex process as reported by Kumar et al. ${ }^{15}$ when evaluating therapeutic options such as AUS device versus Advance $^{\mathrm{TM}}$ male sling (American Medical Systems) for PPI patients. Even if the clinical parameters dictate that AUS maybe a more reasonable option, patient choice dictates otherwise, and this may be contingent on the invasiveness of surgery, desire for a mechanical device, and/or risk to benefit profiling of therapies offered. ${ }^{15}$

In broad terms, if a patient has mild to moderate degree of SUI with some degree of sphincteric function on awake cystoscopy, particularly in a PPI setting, the transobturator Advance ${ }^{\mathrm{TM}}$ male sling (American Medical Systems) is an appropriate choice with good continence rates on intermediate follow-up. Similarly, for moderate to severe SUI, an AUS is the treatment of choice. ${ }^{4}$ There is scant data supporting the use of Advance ${ }^{\mathrm{TM}}$ male sling for patients with severe SUI. ${ }^{16}$

\section{Surgical technique}

Synchronous dual implantation. All dual prostheses/synthetic sling cases require meticulous attention to detail to minimize the risk of infection. At our institution, this commences with sterile preoperative urine cultures and good skin integrity. Operating room is kept at minimum traffic. Intravenous antibiotics consisting of vancomycin and gentamicin is administered $1 \mathrm{~h}$ before incision time. The patients are shaved in the operating room following optimal positioning with slight leg abduction in lithotomy position for sling/IPP or spreader bar supine position for AUS/IPP. A timed 10-min scrub to the genitourinary region with chlorhexidine and alcohol-based disinfectant is performed to decrease intra-operative colonization. Copious antibiotic infused irrigation fluid with gentamicin, amikacin and polymyxin is used throughout the entire case. A 14 French Foley catheter is inserted to drain bladder and facilitate dissection of the urethra. In general, the male sling or AUS device is placed first followed by the IPP. This is to ensure that, in the event of an accidental urethrotomy, surgery can be aborted without discarding the penile prosthesis components.

Male urethral sling/IPP. Rhee et al. ${ }^{17}$ and Christine et al. ${ }^{18}$ have described a two-incision technique for placement of both the

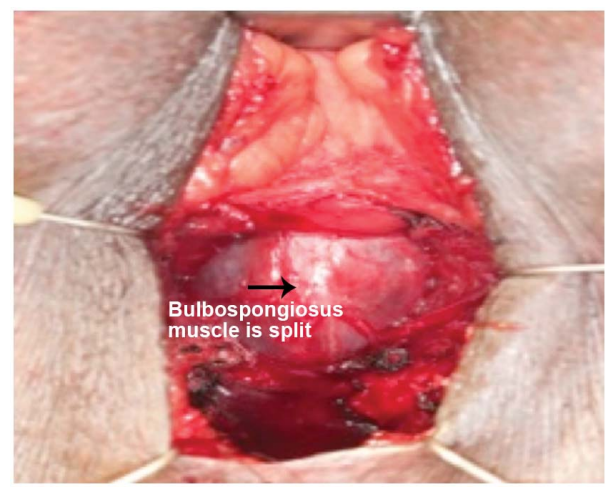

Figure 1 Midline dissection of bulbospongiosus muscle to expose underlying urethra.

InVance $^{\mathrm{TM}}$ or Advance ${ }^{\mathrm{TM}}$ male sling (American Medical Systems) and IPP respectively. A single incision male sling/IPP technique from our institution described by Gorbatiy et al. ${ }^{12}$ is briefly recapitulated. A $5-\mathrm{cm}$ midline perineal incision is made. Dissection is carried to the bulbospongiosus muscle and extended laterally towards the inferior pubic rami. A lone-star retractor with disposable hooks is utilized for surgical exposure. Bone screws attached to looped No. 1 polypropylene suture are inserted into the inner pubic rami: three on each side. The sutures are passed through a trapezoid-shaped synthetic sling and tied down. In the case of Advance ${ }^{\mathrm{TM}}$ sling (American Medical Systems) placement, the bulbospongiosus muscle is split and the central tendon is detached partially (Figure 1). The insertion of the Adductor Longus muscle tendon is identified on either side and a $5-\mathrm{mm}$ stab incision is made $1.5 \mathrm{~cm}$ inferior and $1.5 \mathrm{~cm}$ lateral to this surface landmark (Figure 2). The transobturator fossa is then accessed with the helical trocar via an outside-in approach, exiting at the apex of the dissection between pubic rami and urethra. Once the sling is mounted, care is taken not to place the sling too proximal before tensioning is performed. The expanded central portion of the sling is sutured superficially to the underlying urethra with $4 / 0$ vicryl sutures (Figure 3). Once optimal tensioning is achieved, flexible cystoscopy is performed to confirm proximal urethral advancement and urethral coaptation. The sling arms are retunneled back into the perineal incision and trimmed to promote friction and prevent sling slippage. A new Virtue $^{\mathrm{TM}}$ quadratic sling (Coloplast, Humlebaek, Denmark) that combines the two elements of proximal urethral advancement and distal urethral compression with both a transobturator and prepubic sling

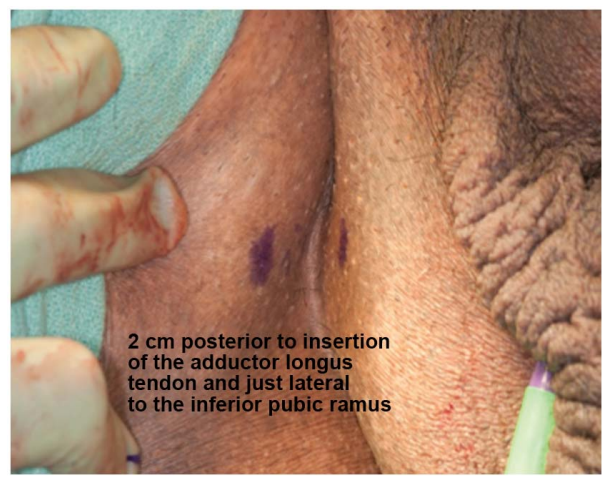

Figure 2 Trocar insertion site $1.5 \mathrm{~cm}$ lateral and inferior to insertion of adductor longus tendon. 


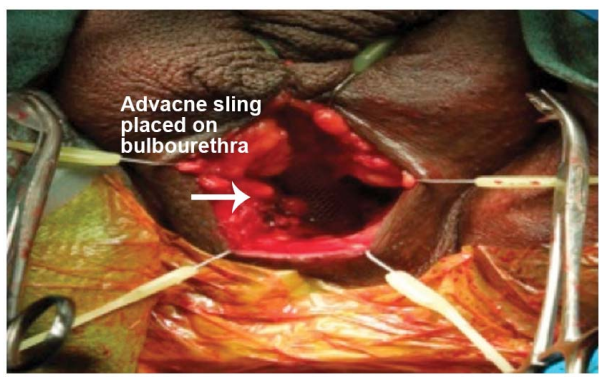

Figure 3 Advance sling secured to urethra with 4/O Vicryl sutures before tensioning.

arms has been studied and early outcomes shows promise although this has not been performed in combination with a penile prosthesis. ${ }^{19}$

In preparation for penile prosthesis implantation, Buck's fascia is cleared to the corpora cavernosa bilaterally. Bilateral $1.5 \mathrm{~cm}$ vertical corporotomies is performed between $2 / 0$ polydioxanone stay sutures (Figure 4). Both corpora may be dilated proximally and distally if necessary with either Furlow inserter or the Dilamezinsert device and the cylinders are sized appropriately (Figure 5). Both corpora are lavaged with copious antibiotic solution before the cylinders are implanted. Note that with a perineal incision, placement of rear tip extenders is rarely required. Correct orientation of the pump and exit tubing must be observed at this point. The corporotomies are closed in a watertight fashion. The fascia transversalis at the selected external inguinal ring medial to the spermatic cord is approached subsequently through the same perineal incision and perforated. The balloon reservoir is placed in the retropubic space of Retzius and filled with sterile normal saline. Ectopic placement of reservoir may be required due to extensive scarring, distorted retropubic anatomy from robotic assisted laparoscopic prostatectomy or previous mesh inguinal hernia repairs. The senior author has previously reported on a novel ectopic placement technique for IPP reservoir placement beneath Scarpa's fascia. ${ }^{20}$

Tubes are connected in the usual fashion using quick connectors. The pump is positioned in the dependent Dartos pouch of the scrotum (Figure 6). The prosthesis is cycled to assess cylinder inflation. Any adjunctive molding for Peyronie's disease can be undertaken at this point while protecting the corporotomy incision. The wound is then closed in two layers. We do not place suction drains on a routine basis but this is generally optional and is surgeon-dependent.

AUS/IPP. A detailed description of the technique for dual AUS/IPP implantation using a single trans-scrotal incision from our institution

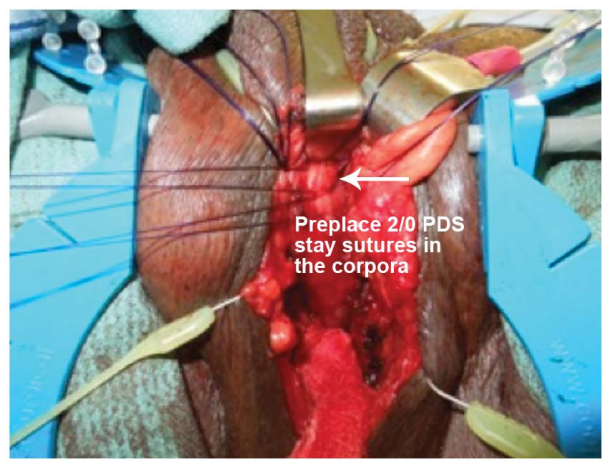

Figure 4 Tunica albuginea exposed and corporotomy between 2/0 polydioxanone stay sutures.

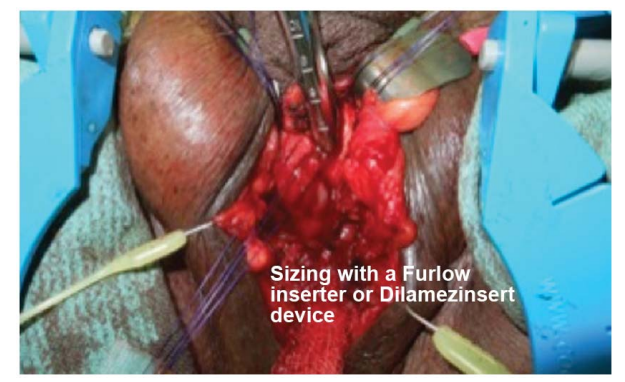

Figure 5 Furlow inserter placed into corpora cavernosum for cylinder implant sizing.

can be referenced to the article by Zafirakis et al. ${ }^{11}$ In brief, meticulous preparation is observed as per sling/IPP approach. A 4-cm single trans-scrotal incision is utilized, although a dual incision can be used (either trans-scrotal or perineal approach). Dissection is carried to the bulbospongiosus muscle where it is split and the corpus spongiosum is identified and dissected as proximal as possible. There has been some debate regarding access to the proximal bulbar urethra with the transscrotal as compared to the perineal approach. Henry et al ${ }^{21}$ reported on a multi-institutional study comparing both routes of implantation and found higher dry rates in the perineal group (44.1\%) compared to the trans-scrotal group $(27.45 \%)(P=0.04)$. Wilson et al. ${ }^{22}$ recently revised his trans-scrotal technique to optimize proximal cuff placement by modifying the $\mathrm{SKW}^{\mathrm{TM}}$ retractor system (American Medical Systems) to facilitate deep bulbar exposure in addition to dissection of the bulbocavernosus muscle prior to cuff placement. The new enhancements to the one-scrotal incision technique allow more proximal cuff placement as evidenced by the bulbocavernosus muscle dissection and use of larger cuffs with comparable continence rate to perineal placed cuffs. ${ }^{22}$ At our institution, we have performed AUS/IPP combination implant using a single perineal incision with good outcomes and we are currently accruing further data for this approach (see Figure 7).

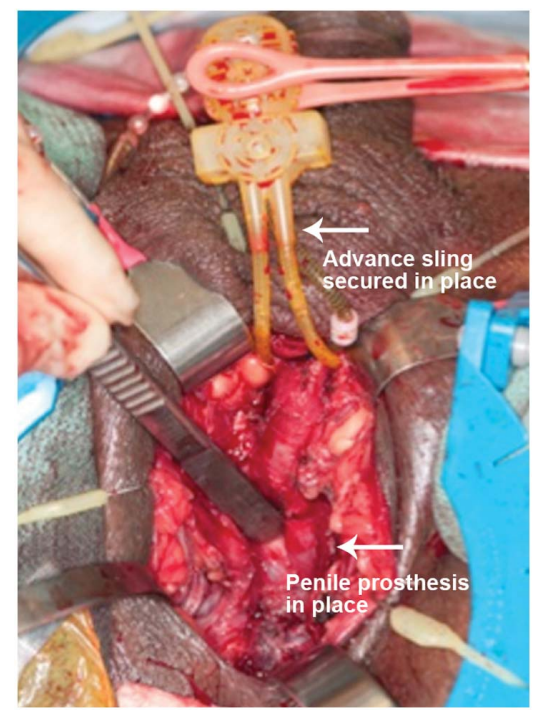

Figure 6 Penile prosthesis cylinders are implanted with corporotomy incisions closed and urethral sling in position before activation pump is placed in dependent portion of scrotum. 


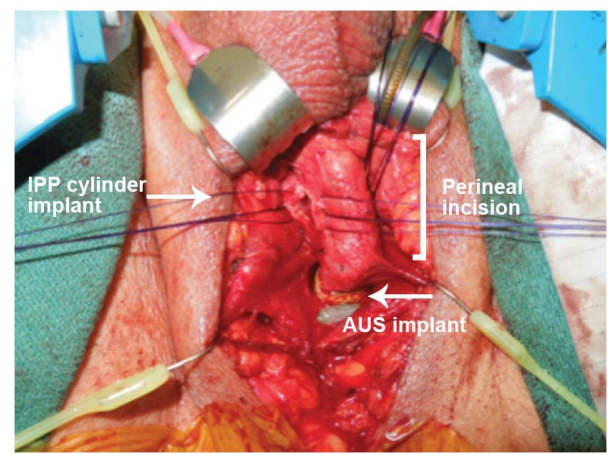

Figure 7 Simultaneous implant of penile prosthesis and artificial urinary sphincter through a single perineal incision.

Dissection is carried posteriorly to the junction between spongiosum and cavernosa and a window is created. The window is enlarged to at least a $1.5-$ to 2 -cm width with right angle clamp to allow American Medical Systems cuff measurement and placement of the cuff. At this juncture, it is appropriate to assess for occult urethral injury by injecting saline in a retrograde fashion. Any leakage at the site of dissection confirms urethral injury and the operation are subsequently terminated.

The pressure regulation balloon is placed retropubically through the designated external inguinal ring after puncturing transversalis fascia. The subdartos pocket is formed and AUS pump is inserted in dependent position on the ipsilateral hemiscrotum. All tubings are connected using the quick-connect system. At this point, the AUS is cycled and locked in the deactivated mode. The penile prosthesis is subsequently implanted as per routine and the reservoir is inserted in the contralateral retropubic space. The tubings are connected to the pump using quick connectors, which are placed in the left hemiscrotum and partially inflated following corporotomy closure. It is important to have tubings for each device compartmentalized separately to avoid cross over. In the event of previous inguinal mesh hernia repairs, a separate lower midline incision may be required to place both balloon reservoirs. A tight circumferential wrap with Kerlix gauze is used for additional hemostasis and prevention of hematoma formation.

All patients are discharged the next operative day following removal of Foley catheter on stool softeners, oral antibiotics and analgesia. Patients return to the clinic in 6 weeks for activation of both prostheses.

\section{Clinical outcomes}

Male sling and IPP. Kaufmann first introduced the concept of urethral compression as a principle of continence control. Since that time, research and development have led to gradual evolution of sling products design for SUI treatment in men. ${ }^{10}$ Bulbourethral sling is an attractive option for men with mild to moderate SUI due to its minimally invasive nature, immediate efficacy and low morbidity. It also overcomes the mechanical disadvantages of an AUS. There are three classes of bulbourethral sling systems: (i) the bone anchored slings (BAS); (ii) the transobturator system; and (iii) the re-adjustable sling system. The latter is not currently available in the United States.

The first modern sling was the bone anchor perineal InVance sling, which was based on the principle of providing ventral urethral compression of the bulbar urethra to obtain a functional non-obstructive seal. The sling was first introduced by Madjar et al. ${ }^{23}$ and Comiter et al. ${ }^{24}$ with reported cure rates ranging between $58 \%$ and $86 \%$. The complication rates were reported between $0 \%$ and $14.5 \%$ with recurrent SUI and osseous pain. On intermediate follow-up of 48 months duration, the continence and improvement rates remained at $65 \%$ and $20 \%$, respectively. ${ }^{24}$ Styn et al..$^{25}$ recently reported on their BAS cohort of 119 men who were predominantly men with PPI. Continence rates were in the order of $74.8 \%$ over a median follow-up duration of 13 months and higher complication rates than previous were reported. There was an overall $58.8 \%$ complication rate with a reoperation rate of $26.9 \%$ for the index case. Recurrent SUI being commonest $(25.2 \%)$ is followed by urge urinary incontinence $12.6 \%$ and infection $16 \%$, respectively. There were no osseous related complications. ${ }^{25}$

Rehder and Gozzi ${ }^{5}$ subsequently pioneered the transobturator Advance $^{\mathrm{TM}}$ sling (American Medical Systems) based on the concept of restoring the continence mechanism by advancing the proximal urethra to provide urethral coaptation in men with residual sphincteric function. Cornu et al. ${ }^{26,27}$ reported from their prospective study the intermediate outcomes on Advance ${ }^{\mathrm{TM}}$ sling (American Medical Systems) in 136 patients with mild to moderated incontinence. They reported a cure and improved continence rates of $65 \%$ and $16 \%$ respectively at a mean follow-up of 21 months and highlighted three prognostic factors leading to poorer outcomes which included: (i) previous radiotherapy; (ii) pad weight $>200 \mathrm{gm} / 24 \mathrm{~h}$; and (iii) history of interval stricture disease before sling implantation. More recently, Rehder et al. ${ }^{6}$ reported on their 3 -year outcome from a multi-institutional study with 156 patients in a prospective fashion and reported durability of effect with $76.8 \%$ either cured or improved from their incontinence. The only independent predictor of success in multivariate analysis was preoperative pad use $(P=0.036)$.

Rhee $^{17}$ reported the first small series of combined InVance ${ }^{\mathrm{TM}}$ sling (American Medical Systems) and penile prosthesis for concurrent treatment of ED and SUI using a two-incision technique (perineal and transscrotal). He reported $100 \%$ efficacy in continence rates with good restoration of erections with no adverse events in a mean follow-up duration of 15 months. Subsequently Gorbatiy et al. ${ }^{12}$ described the first series of combination surgery using InVance ${ }^{\mathrm{TM}}$ or Advance ${ }^{\mathrm{TM}}$ male sling (American Medical Systems) and IPP via a single perineal incision. They reported cost benefits and clinical outcomes in eight patients (three InVance and five Advance male sling) with mean follow-up of 13.6 months. All men had excellent post-operative Sexual Health Inventory for Men scores with significant decrease in pad use to mean of 1 pad. In addition, patients with dual implants were able to save US\$9000 on average. One patient developed urinary retention requiring catheterization. No complications with erosion or infection were reported. Cornu et al. ${ }^{27}$ reported on their initial experience combination transobturator male sling and penile implant after radical prostatectomy in four patients (two synchronous and two sequential implants) with $100 \%$ continence and high satisfaction in sexual function. Christine et al. ${ }^{18}$ reported on their intermediate outcomes in their combination series with a twoincision technique and reported continence cure rate of $86 \%$ and a sexual function satisfaction of $89 \%$ at a mean of 22 months follow-up. The same author also further reported on an infection rate of $1.2 \%$ in their series of 78 men at mean of 16 months follow-up. ${ }^{28}$ The above studies demonstrate good safety and efficacy of dual synchronous implantation with short to intermediate term follow-up.

AUS and IPP. Both AUS and IPP are the current gold standards for treatment of severe SUI and ED respectively. Numerous studies centered on single implant series have reported good objective cure/ improvement continence rates and high patient satisfaction with sexual function at long-term follow-up. ${ }^{29}$ In terms of synchronous dual implantation, there is a lack of good quality data. Most are either part 
of intra-series reports on outcomes focused primarily on single prosthesis series with short-term follow-up from specialized tertiary referral centers. The earliest report comes from Graham et al. ${ }^{10}$ with a multiple incision technique. They reported almost a $100 \%$ reoperation rate using the Kaufmann prosthesis. Perhaps the biggest detractor to synchronous dual prostheses implantation is the concern of infection with the need for explantation of all prostheses components and difficult access for subsequent prosthesis placements. This claim however lacks scientific backing as much of the adverse outcomes were reported using the earlier model prosthesis. With new refinements in the design of the prosthetic devices, the introduction of antibiotic retardant coating, and improvements in surgical technique and surgeon expertise, there is a renewed interest in this approach.

Parulkar and Barrett ${ }^{30}$ reported their outcomes with dual synchronous implants in 40 out of 65 patients with various prostheses devices (AMS 721, AMS 800, semirigid rods to AMS 700CX; American Medical Systems) via a two-incision approach. With a mean followup of 36 months they reported continence rates of $95 \%$ ( 1 pad or less) and $98 \%$ of the IPP was functional. There was an average re-operation rate of 0.98 corrections per patient, with the AUS doubling the IPP revisions. The overall erosion/infection rate was $11 \%$. Although no further subset analysis was performed, patients with AMS 800 (25\% versus $62 \%$ with earlier models) and AMS 700 CX prosthesis performed better with less revision surgeries.

Wilson et al. ${ }^{9}$ in their initial study using the trans-scrotal technique reported on 12 patients with concurrent implantation of three-piece IPP and AUS (AMS 800) in their study of 37 patients undergoing AUS placement. Nine patients were virgin dual implants with the rest indicated for AUS revision surgery and concurrent IPP. They reported continence cure rate of $66 \%$ and improvement of $34 \%$ respectively with mean follow-up of 12 months. The AUS erosion rate was $8 \%$ with one case of urethral injury intra-op and two cases likely iatrogenic occurring within 6 weeks post operatively. There was one IPP infection requiring explantation but the AUS was salvaged. Although there were five patients who were irradiated, these were not distinguished amongst the reported patients with adverse outcome. Similarly outcomes were not distinguished between the synchronous and sequential implant group.

Kendirci et al. ${ }^{31}$ reported on a multicenter retrospective study involving 22 post-prostatectomy patients with dual synchronous implants (AMS 700CX IPP and AMS 800) via a trans-scrotal approach. At 17 months follow-up $100 \%$ of the patients were significantly improved requiring one pad or less with an overall revision rate of $14 \%$ with two patients developing AUS cuff erosion and one patient for reservoir migration.

Sellers et al. ${ }^{32}$ reported the cost and time benefit analysis in their prostheses series of 15 patients with dual implants in a series of 128 men who underwent prosthesis implantation (92 IPP, 21 AUS and 15 dual prostheses). Dual prosthesis implantation time was decreased by $24.7 \%$ compared with total time for individual prosthesis, with cost savings of USD7000 as compared to staged implants. At 16 months, they reported no erosion or infections although functional outcomes were not reported.

Mancini et al..$^{33}$ presented patient satisfaction outcomes in 95 men of which 33 patients had dual prosthesis implant at a mean follow up of 21.6 months. The overall satisfaction rate was $94 \%$ on telephone interview with standardized questionnaire evaluation, with $87 \%$ to $94 \%$ reporting recommending dual implant to a friend or relative. There was a $76 \%$ reduction in pad use from mean of 6.1 to 1.3 pads per day. Interestingly, the rigidity for penetration in the dual implant group was better than the IPP alone group (97\% versus $87.1 \%$ ) although not statistically significant. Revision rates between dual implants and single prosthesis implants were comparable in their series. Table 1 summarizes the published data available on combination surgery to date.

What about AUS failures? AUS failures have been shown by Christine et $a l^{34}$ to be salvaged successfully with Advance sling without explanting the sphincter. No technical modification was required for sling placement. The pad use for recurrent SUI was $2-5$ pads post AUS failure. Mean time from AUS implant to salvage sling was 32 months. AUS had been implanted via both perineal and trans-scrotal approaches. Early results with mean of 13 months follow-up in 19 men were encouraging with $79 \%$ dry rate (defined as no pad use) and $21 \%$ social continence (defined as 1 pad per day).

\section{Pro-ACT device}

There is no published data available on combination therapy with IPP and Pro-ACT device. Amongst the newer generation of products released to circumvent some of the issues with the AUS is the ProACT device (Uromedica Inc.) currently in Phase III trials awaiting FDA approval. The device consists of a silicone balloon on the proximal end and titanium port in the distal end. It is inserted transperineally under fluoroscopy or transrectal ultrasound to the level of the urethrovesical anastomosis bilaterally. Surgical technique has been previously published. ${ }^{35-37}$ Currently there are no publications relating to combination surgery with this device with penile prosthesis, but given that access is gained via a transperineal route, this can easily be incorporated. Mechanical compression of urethra is achieved upon balloon inflation to restore outlet resistance. The advantages of the device include minimally invasive technique, lack of circumferential compression, as well as being adjustable post-operatively with titratable volume injections delivered through the titanium ports implanted superficially in the scrotum.

Table 1 Combination surgery for stress urinary incontinence and erectile dysfunction

\begin{tabular}{|c|c|c|c|c|c|c|}
\hline & & IPP device & No. of patients & Follow-up (months) & SHIM/function & Post-op pad use \\
\hline IPP and male slings & SUI device & & & & & \\
\hline Rhee et al., $2005^{17}$ & InVance & AMS 700 & 4 & 15 & NA/satisfactory & $0(100 \%)$ \\
\hline Gorbatiy et al., $2010^{12}$ & InVance/Advance & AMS 700 & $8(3 / 5)$ & 13.6 & 23.5 & $1(100 \%)$ \\
\hline Parulkar et al., $1989^{30}$ & AMS792/800 & Various & 40 & 35.7 & NA/98\% functional & $0-1(90 \%)$ \\
\hline Wilson et al., $2003^{9}$ & AMS 800 & NA & 12 & 12 & NA & $0-1(100 \%)$ \\
\hline Seller et al., $2004^{32}$ & AMS 800 & AMS700CX & 15 & 16 & NA & NA \\
\hline
\end{tabular}

Abbreviations: AMS (American Medical Systems, Minnetonka, MN, USA); AUS, artificial urinary sphincter; IPP, inflatable penile prosthesis; SHIM, Sexual Health Inventory for Men; SUI, stress urinary incontinence. 
Hubner et al. ${ }^{35}$ first reported on a prospective trial of 117 men with SUI-95\% were post radical prostatectomy. With a short term followup of 13 months they reported a cure rate of $67 \%$ with $92 \%$ significant improvement and an average of 3 balloon volume adjustments. However in their report there was a substantial decrease in response rate and a corresponding $36 \%$ of patients reporting unsatisfactory outcome and $21 \%$ proceeded to have an AUS.

A multicenter pilot study trial by Lebret et al. ${ }^{36}$ reported $30 \%$ cure rate and 59\% were improved at 12 months and with $31 \%$ complication rate. Recent studies suggest that this option is less applicable for severe SUI or patients with prior irradiation. Gilling et al. ${ }^{38}$ published their initial small series of 37 patients mainly post-radical prostatectomy radiotherapy naive with $81 \%$ of men requiring one pad or less at minimum 24 months follow-up. The reported complication however is high with infection rates of $9 \%$. Longer follow-up is required to ascertain its role in the management of male SUI.

\section{CONCLUSION}

Combination surgery for the treatment of medically refractory ED and SUI appears to be a viable option for an increasing population men with concurrent morbidity generated from our surgical interventions. It is technically feasible with either a single or a double incision approach. Penile prosthesis and AUS device implants have consistently been associated with high satisfaction rates and excellent durability. Simultaneous implantation is finding new favor with urologist in view of potential patients benefits with comparable functional and adverse outcomes compared to single implant series. For patients with lesser degree of urinary incontinence, the Advance ${ }^{\mathrm{TM}}$ male sling (American Medical Systems) have demonstrated safety and durability of continence rate on short to intermediate-term follow-up either in isolation or in combination with penile prosthesis. All patients should be screened and considered for combination surgery where applicable, to achieve the ultimate trifecta of local cancer control and restoration of organ function. The need for early referral for combination management in the convalescent phase, preferably in a dedicated comprehensive unit with subspecialty interests is recommended.

\section{COMPETING FINANCIAL INTERESTS}

The authors declare no competing financial interests.

1 Dubbelman YD, Dohle GR, Schröder FH. Sexual function before and after radical retropubic prostatectomy: a systematic review of prognostic indicators for a successful outcome. Eur Urol 2006; 50: 711-8; discussion 718-20.

2 Penson DF, McLerran D, Feng Z, Li L, Albertsen PC et al. 5-year urinary and sexual outcomes after radical prostatectomy: results from the prostate cancer outcomes study. J Urol 2005; 173: 1701-5.

3 Lai HH, Hsu EI, Teh BS, Butler EB, Boone TB. 13 years of experience with artificial urinary sphincter implantation at Baylor College of Medicine. J Urol 2007; 177: 1021-5.

4 Gousse AE, Madjar S, Lambert MM, Fishman IJ. Artificial urinary sphincter for postradical prostatectomy urinary incontinence: long-term subjective results. J Urol2001 166: $1755-8$

5 Rehder P, Gozzi C. Transobturator sling suspension for male urinary incontinence including post-radical prostatectomy. Eur Urol 2007; 52: 860-6.

6 Rehder P, Haab F, Cornu JN, Gozzi C, Bauer RM. Treatment of postprostatectomy male urinary incontinence with the transobturator retroluminal repositioning sling suspension: 3-year follow-up. Eur Urol 2012; 62: 140-5.

7 Bettocchi C, Palumbo F, Spilotros M, Lucarelli G, Palazzo S et al. Patient and partne satisfaction after AMS inflatable penile prosthesis implant. J Sex Med 2010; 7(1 Pt 1): 304-9.

8 Eid JF, Wilson SK, Cleves M, Salem EA. Coated implants and 'no touch' surgica technique decreases risk of infection in inflatable penile prosthesis implantation to $0.46 \%$. Urology 2012; 79: 1310-5

9 Wilson SK, Delk JR 2nd, Henry GD, Siegel AL. New surgical technique for sphincter urinary control system using upper transverse scrotal incision. J Urol 2003; 169 261-4.
10 Graham SD Jr, Carson CC 3rd, Anderson EE. Long-term results with the Kaufman prosthesis. J Urol 1982; 128: 328-30.

11 Zafirakis $\mathrm{H}$, Wang R, Westney OL. Combination therapy for male erectile dysfunction and urinary incontinence. Asian J Androl 2008; 10: 149-54.

12 Gorbatiy V, Westney OL, Romero C, Wang R. Outcomes of simultaneous placement of an inflatable penile prosthesis and a male urethral sling through a single perineal incision. J Sex Med 2010; 7(2 Pt 1): 832-8.

13 Lebret $\mathrm{T}$, Cour F, Benchetrit J, Grise P, Bernstein J et al. Treatment of postprostatectomy stress urinary incontinence using a minimally invasive adjustable continence balloon device, ProACT: results of a preliminary, multicenter, pilot study. Urology 2008; 71: 256-60.

14 Ghanaat M, Tsui J, Blaivas J, Shah M, Weiss J et al. Does pad usage reflect the severity of urinary incontinence? J Urol 2011; 185 (Suppl 4): e464.

15 Kumar A, Litt ER, Ballert KN, Nitti VW. Artificial urinary sphincter versus male sling for post-prostatectomy incontinence-what do patients choose? J Urol 2009; 181: 1231-

16 Vella M, Romeo S, Leto G, Salamone C, Melloni D. Treatment of severe postprostatectomy stress urinary incontinence using AdVance sling. Urologia 2011; 78 67-71. Italian

17 Rhee EY. Technique for concomitant implantation of the penile prosthesis with the male sling. J Urol 2005; 173: 925-7.

18 Christine B, Wilson SK, Shamloul R, Bella AJ. Simultaneous placement of an inflatable penile prosthesis and advance male sling for erectile dysfunction and incontinence: robust efficacy and safety data at 2 year follow-up. J Urol 2010; 183 (Suppl 4): e490.

19 Comiter CV, Nitti V, Elliot C, Rhee E. A new quadratic sling for male stress incontinence: retrograde leak point pressure as a measure of urethral resistance. J Urol 2012; 187: 563-8.

20 Wang R, Lin HC. Sub-Scarpa's facia placement of the penile prosthesis reservoir: new surgical technique. J Sex Med 2010; 7 (Suppl 4): 180; abstract No. 085.

21 Henry GD, Graham SM, Cornell RJ, Cleves MA, Simmons CJ et al. A multicenter study on the perineal versus penoscrotal approach for implantation of an artificial urinary sphincter: cuff size and control of male stress urinary incontinence. J Urol 2009; 182: 2404-9.

22 Wilson SK, Aliotta PJ, Salem EA, Mulcahy JJ. New enhancements of the scrotal oneincision technique for placement of artificial urinary sphincter allow proximal cuff placement. J Sex Med 2010; 7: 3510-5.

23 Madjar S, Jacoby K, Giberti C, Wald M, Halachmi S et al. Bone anchored sling for the treatment of post-prostatectomy incontinence. J Urol 2001; 165: 72-6.

24 Comiter CV. The male perineal sling: intermediate-term results. Neurourol Urodyn 2005; 24: 648-53.

25 Styn NR, McGuire EJ, Latini JM. Bone-anchored sling for male stress urinary incontinence: assessment of complications. Urology 2011; 77: 469-73.

26 Cornu JN, Sèbe $P$, Ciofu C, Peyrat L, Cussenot $O$ et al. Mid-term evaluation of the transobturator male sling for post-prostatectomy incontinence: focus on prognostic factors. BJU Int 2011; 108: 236-40.

27 Cornu JN, Chanu T, Beley S, Sèbe P, Peyrat Let al. Initial experience combinating transobturator male sling and penile implant after radical prostatectomy. Prog Urol 2011; 21: 349-53.

28 Christine B, Knoll LD. Simultaneous advance male sling and an inflatable penile prosthesis: concurrent placement does not increase potential for implant infection. J Urol 2011; 185 (Suppl 4): e726.

29 Carson CC 3rd, Mulcahy JJ, Harsch MR. Long-term infection outcomes after original antibiotic impregnated inflatable penile prosthesis implants: up to 7.7 years of follow up. J Urol 2011; 185: 614-8.

30 Parulkar BG, Barrett DM. Combined implantation of artificial sphincter and penile prosthesis. J Urol 1989; 142: 732-5.

31 Kendirci M, Gupta S, Shaw K, Morey A, Jones L et al. Synchronous prosthetic implantation through a transscrotal incision: an outcome analysis. J Urol 2006; 175: 2218-22.

32 Sellers CL, Morey AF, Jones LA. Cost and time benefits of dual implantation of inflatable penile and artificial urinary sphincter prosthetics by single incision. Urology 2005; 65: 852-3.

33 Mancini JG, Kizer WS, Jones LA, Mora RV, Morey AF. Patient satisfaction after dua implantation of inflatable penile and artificial urinary sphincter prostheses. Urology 2008; 71: 893-6.

34 Christine B, Knoll LD. Treatment of recurrent urinary incontinence after artificia urinary sphincter placement using the AdVance male sling. Urology 2010; 76 1321-4.

35 Hübner WA, Schlarp OM. Treatment of incontinence after prostatectomy using a new minimally invasive device: adjustable continence therapy. BJU Int 2005; 96: 58794.

36 Lebret T, Cour F, Benchetrit J, Grise P, Bernstein J et al. Treatment of postprostatectomy stress urinary incontinence using a minimally invasive adjustable continence balloon device, ProACT: results of a preliminary, multicenter, pilot study. Urology 2008; 71: 256-60.

37 Gregori A, Romanò AL, Scieri F, Pietrantuono F, Incarbone GP et al. Transrecta ultrasound-guided implantation of adjustable continence therapy (ProACT): surgical technique and clinical results after a mean follow-up of 2 years. Eur Urol 2010; 57: 430-6.

38 Gilling PJ, Bell DF, Wilson LC, Westenberg AM, Reuther R et al. An adjustable continence therapy device for treating incontinence after prostatectomy: minimum 2-year follow-up. BJU Int 2008; 102: 1426-30; discussion 1430-1. 